Communications in Physics, Vol. 19, No.1 (2009), pp. 1-8

\title{
TIGHT-BINDING CALCULATIONS OF BAND STRUCTURE AND CONDUCTANCE IN GRAPHENE NANO-RIBBONS
}

\author{
HOANG MANH TIEN, NGUYEN HAI CHAU \\ Institute of Physics, VAST \\ PHAN THI KIM LOAN \\ Physics Department, School of Education, Can Tho University
}

\begin{abstract}
We suggest a general approach based on the nearest-neighbor tight-binding approximation (TB) to investigate the band structure and conductance of a quasi-one dimensional system. Numerical calculations carried out for Graphene nanoribbons (GNRs) with different widths and edge conditions (zigzag and armchair) reveal the well-known results that the electronic properties of GNRs depend strongly on the size and geometry of the sample.
\end{abstract}

Although various carbon-based structures have been studied for decades, graphene was only isolated experimentally in 2004 [1,2]. Graphene, the only truly two-dimensional (2D) nano crystal, is made out of carbon atoms arranged on the honeycomb structure. Recently, graphene has attracted much attention, both experimental and theoretical due to its unique and unusual electronic properties. One of the most interesting features is that, low energy excitations are massless, chiral, Dirac fermions which mimic the relativistic particles [1,2], though its Fermi velocity is 300 times smaller than the speed of light c $\left(v_{F} \approx 10^{6} \mathrm{~ms}^{-1}\right)$. In addition, the density of material electrons may be huge, $n_{e} \approx$ $4 \times 10^{15} \mathrm{~cm}^{-2}$ [3] that makes the graphene look the most promising for nanoelectronic devices in the future.

The fact that distinguishes graphene from ordinary semi-conductantor and metallic systems, the Dirac fermions do not obey the conventional Schrödinger equation. In 1984, based on the $\vec{k} \cdot \vec{p}$ approximation, Vincenzo and Mele found that the low energy excitations obey the two-dimensional Dirac-like equation [5]. Solving this equation, one can find out the wave functions of fermions and other dynamic properties of infinite graphene sheets $[1,2,4]$. However, in experiment and technology, GNRs ( narrow graphene strips) are more attractive because of their potential in creating nanoelectronic devices. In fact, to deal with GNRs one can also use the Dirac-like equation approach with appropriate boundary conditions to $[1-3,6,7]$. Nevertheless, when the GNRs become narrower (the width of the GNRs is less than $15 \dot{\mathrm{A}}$ ) the results of the $\vec{k} \cdot \vec{p}$ approximation do not work well due to the edge effects [6]. In this case, the tight-binding (TB) approximation is a more effective method because of its more accuracy. There are numerous papers in which the TB method is used for GRNs with the different geometries and sizes [8-11]. In this paper, we suggest a general approach based on the TB method to calculate band structure and conductance 
of a quasi-one dimensional (Q1D) structure. In order to explicitly illustrate this method, we bring out the numerical results for the band structure and conductance of the GNRs with two well-addressed types of edges, zigzag and armchair. Our results are qualitatively in agreement with those obtained from other methods. Detailed calculations for the band structure show that all zigzag GNRs are metallic with localized states on two edges, while armchair GNRs are either metallic or insulating, depending on the ribbon widths $[6,10,12$ 16]. Numerical analyses of the conductance also demonstrate that due to the finite width and geometry of GNRs the conductance will be quantized [3,7,17-19], increasing step by step as Fermi energy increases.

Let us consider a quasi-one dimensional structure built up from unit cells of $N_{0}$ sites. Base on tight-binding approximation, the Schrodinger equation takes the form:

$$
H_{n, n-1} \psi_{n-1}+H_{n, n} \psi_{n}+H_{n, n+1} \psi_{n+1}=E \psi_{n},
$$

where $\psi_{n}$ is the $N_{0}$-dimensional vector representing the wave function at $n^{\text {th }}$ unit cell, Hamiltonian $H_{n, m}(m=n, n \pm 1)$ is a $N_{0} \times N_{0}$ matrix. It is worth to notice that only the nearest neighbor interaction terms of the Hamiltonian appear in the expression . We can obtain, due to periodicity of the structure: $H_{n, n}=H_{0}, H_{n, n-1}=H_{1}$ and $H_{n+1, n}=H_{2}$ (Hermite conjugation requires that $H_{2}=H_{1}^{+}$). To solve the equation (1), based on Bloch's theorem, we seek the solution in the form $\psi_{n} \sim e^{i k n}$, where $k$ is the Bloch wave vector. By inserting this form of the solution into the equation (1), the simple eigenequation for eigenvalue $E$ is brought out:

$$
\operatorname{det}\left(H_{1} e^{-i k}+H_{0}+H_{2} e^{i k}-E I\right)=0 .
$$

The equation (2) gives $N_{0}$ solutions $\mathrm{E}(\mathrm{k})$ corresponding $N_{0}$ subbands of the dispersal relation. In the case of Garaphene, one will see that a half of them is valent, and the rest is conductive.

Next, we will derive the formula for the conductance of a quasi-one dimensional structure. In order to do that we consider a sample sandwiched between the two semiinfinite leads. It is assumed that two semi-infinite leads have the same lattice structures as that in the centre sample to avoid any scattering contribution from the mismatched interfaces between different types of lattice. The leads are doped so much that make them nearly metallic, while the sample may be lighter doped, or maybe imposed by some extrafields. Due to the extra-fields, the periodicity is broken inside the sample that contributes to the different form of the Hamiltonian in side the sample. The Hamiltonian $H_{n, m}$, which represents for the interaction between $n^{t h}$ and $m^{\text {th }}$ unit cells, will be changed to different forms due to concrete kinds of doping or particular extra-fields. The Landauer-Buttiker formula for conductance is given by [20]:

$$
G=\frac{2 e^{2}}{\hbar} \sum_{n} \sum_{m} \frac{I_{m}}{I_{n}}\left|t_{m n}^{2}\right|,
$$

where $t_{m n}$ is the transmission coefficient of the incoming wave in $n^{\text {th }}$ band on the left and the outgoing wave in $m^{\text {th }}$ band on the right, $I_{n}$ and $I_{m}$ are the corresponding currents. 
Conventionally, the probability current is proportional to the group velocity which is derived as: $v_{g}=d E / d p$. We notice that base on the general tight-binding Hamiltonian, the probability current can be found by the original procedure of quantum mechanics, which gives:

$$
I_{n, n-1} \sim \psi_{n}^{\dagger} H_{1} \psi_{n-1}-\psi_{n-1}^{\dagger} H_{1}^{\dagger} \psi_{n} .
$$

For Bloch state in band $q$ of energy $E$ we simply have to insert $\psi_{n}=C^{q}(E) e^{i k^{q} n}$ into the equation (4) to gain the probability current of the state.

In the next part, we will use the TB approach to calculate the transmission coefficients $t_{m n}$. It has simply been obtained by solving the Schrodinger equation for scattering states, mathematically, the equation (1) will be solved with appropriate boundary condition. Roughly speaking, boundary of scattering states is physically described as: the wave function injects from the left lead purely in the band $p$ and then it is scattered into outgoing (from the sample) wave functions in all the bands. The wave functions in the left and right leads take the form:

$$
\psi_{n}=C^{p}(E) e^{i k^{p} n}+\sum_{q \in Q_{1}, Q_{10}} r_{q p} C^{q}(E) e^{i k^{q} n},
$$

for the left lead and

$$
\psi_{n}=\sum_{q \in Q_{2}, Q_{20}} t_{q p} C^{q}(E) e^{i k^{q} n},
$$

for the right lead. In which, for each energy $E$, the wave numbers $k^{q}(E)$, derived from dispersal equation (2), are divided in to four sub-sets: $Q_{1}$ for left to right wave, $Q_{10}$ left to right decay wave, $Q_{2}$ right to left wave, $Q_{20}$ right to left decay wave.

These conditions can be viewed as boundary conditions for the wave function in the sample. In fact, imposing them in the general form on the wave equation [1] of the sample, we will gain the formulae for $t_{q p}$ and $r_{q p}$, this can be called matching the wave function through the sample. To visualize how the wave function will be matched, we have to solve the tight-binding equation (1) for all the sites in the sample (note the periodicity is now broken) with coupling to the wave function on the leads.

The equation (1) for $n$ altering from 0 to $N+1$ can be viewed as $(N+1) \times N_{0}$ linear equations for the unknown $\psi_{n}$ while both $\psi_{-1}$ and $\psi_{N+2}$ are considered to be known. It is worth to notice that for $n=0$ and $n=N+1$ the wave functions belong to the leads rather than to the sample. The equation can be written down in the matrix form: $A *\left(\psi_{0}, \psi_{1}, \ldots, \psi_{N+1}\right)^{t}=\left(-H_{0,-1} \psi_{-1}, 0, \ldots,-H_{N+1, N+2} \psi_{N+2}\right)^{t}$, in which $\mathrm{A}$ is the coefficient matrix which can be expressed explicitly:

$$
A=\left(\begin{array}{ccccc}
H_{0,0}-E & H_{0,1} & \cdots & 0 & 0 \\
H_{1,0} & H_{1,1}-E & \cdots & 0 & 0 \\
0 & H_{2,1} & \cdots & 0 & 0 \\
. & \cdot & \cdots & \cdot & \cdot \\
0 & 0 & \cdots & H_{N, N}-E & H_{N, N+1} \\
0 & 0 & \cdots & H_{N+1, N} & H_{N+1, N+1}-E
\end{array}\right)
$$



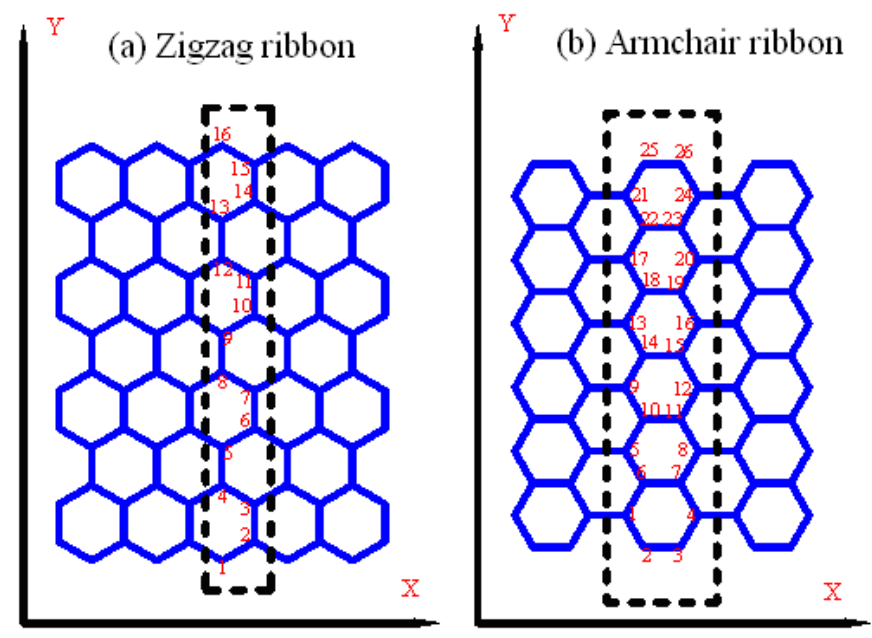

Fig. 1. Schematic geometry of a unit cell, which has $N_{0}$ atoms, in the tightbinding model used. The unit cells are chosen corresponding to the two types of edges: (a) the zigzag edge and (b) the armchair edge.

Solving the equation we have the solution in the form $\left(\psi_{0}, \psi_{1}, \ldots, \psi_{N+1}\right)^{t}=G_{0} *$ $\left(-H_{0,-1} \psi_{-1}, 0, \ldots,-H_{N+1, N+2} \psi_{N+2}\right)^{t}$, where $G_{0}=A^{-1}$. Note that we need to match up the wave function between the leads and the sample, so extracting only the term of wave function on the lead we have the relation in the form $\left(\psi_{0}, \psi_{N+1}\right)^{t}=\bar{G}\left(-H_{0,-1} \psi_{-1}\right.$,

$\left.-H_{N+1, N+2} \psi_{N+2}\right)^{t}$. Inserting the "forms" (5) and (6) into this equation, one have the equations for $t_{q p}$ and $r_{q p}$.

The above argument is so general that this method can be utilized for any periodic Q1D structures. The difference between particular systems is related to the widths and edge conditions which are represented in the Hamiltonian. For a particular system, it is not very difficult to write the Hamiltonian down explicitly because nearest neighbor interactions are only considered. The dispersal relation is calculated by using equation (2). The probability current can be inferred from equation (4) and the transmission coefficients are computed as we show above. After that we can easily calculate the conductance, using equation (3). Obtained numerical results are presented in Fig 2-4 for GNRs with zigzag and armchair edges and with different widths.

GNRs are cut out from the graphene sheet, the honey-comb lattices of Carbon atoms on a plane $[1,2]$, along a direction with a defined width. As carbon nanotubes, the ribbons may have various geometrical form due to the direction of "chiral", among them, the most common types being of interest are armchair-edge and zigzag-edge ribbons [2]. The geometry of these GNRs are illustrated on the top and bottom edges of Fig. 1. In the zigzag case (Fig. 1a), it is interesting that the atoms at each edge are of the same sublattice (A on the top and B on the bottom edge). In this figure, we also show the unit cell used in the tight-binding calculation for the zigzag ribbons, containing $N_{0} / 2$ A-type atoms that 




Fig. 2. The band structures for a zigzag graphene nanoribbon, $E(t)$ versus $k a_{0}$, $k$ is the wave vector parallel to the graphene nanoribbon edges, $a_{0}$ is the lattice constance, $t$ is the nearest neighbor hopping energy.

alternate along the unit cell with $N_{0} / 2$ B-type atoms. The total width of the GNRs is $W=N_{0} / 4 * \sqrt{3}, a_{0}-a_{0} / \sqrt{3}$, where $a_{0}$ is lattice constant, $a_{0}=\sqrt{3} a_{c c}\left(a_{c c}=1.42 \dot{\mathrm{A}}\right.$, the C-C bond length). Similarly, the structure of the armchair GNRs consists of atoms of two types of sublattices A and B as illustrated on the Fig. 1b. Each unit cell also contains $N_{0} / 2$ A-type atoms and $N_{0} / 2$ B-type atoms alternately. However, it is different from the zigzag edge that in each edge (top or bottom), there have both A-type atoms and B-type atoms alternately and the total width is $W=\left(N_{0}-2\right) / 4 * a_{0}$.

In Fig. 2 and Fig. 3, the band structures in zigzag and armchair edges are presented. It is explicitly that the spectrum breaks into a set of subbands due to the quantized confinement. In Fig. 2, the conductance of zigzag GNRs with 88 atoms per unite cell (approximately $10 \mathrm{~nm}$ width) is depicted. In fact, the lowest conduction band and the upmost valence band touch at the Dirac points, that leads to the metallic behavior. These gapless bands produced confinement of the electronic states near the Dirac points [1]. The same result was also obtained for the GNRs with different widths $N_{0}=86,92$. And therefore we arrived at the well known result that all zigzag GNRs are metallic $[6,12,13]$. The GNRs with armchair edge of 88 (a) and 90 (b) atoms per unit cell are considered; the band structures are showed in the Fig. 3. As can be seen in this figure, the band structures of the armchair GNRs depend strongly on their widths. Due to the gap between conduction and valence band, the armchair GNRs can be metallic (the gapless case, Fig. 3a) or semiconductor (the gapped case, Fig. 3b). It means that armchair GNRs are metallic when the number of atoms in unit cell: $N_{0}=3 n+1$ ( $n$ is an integer), and insulating otherwise $[1,3,6]$. 


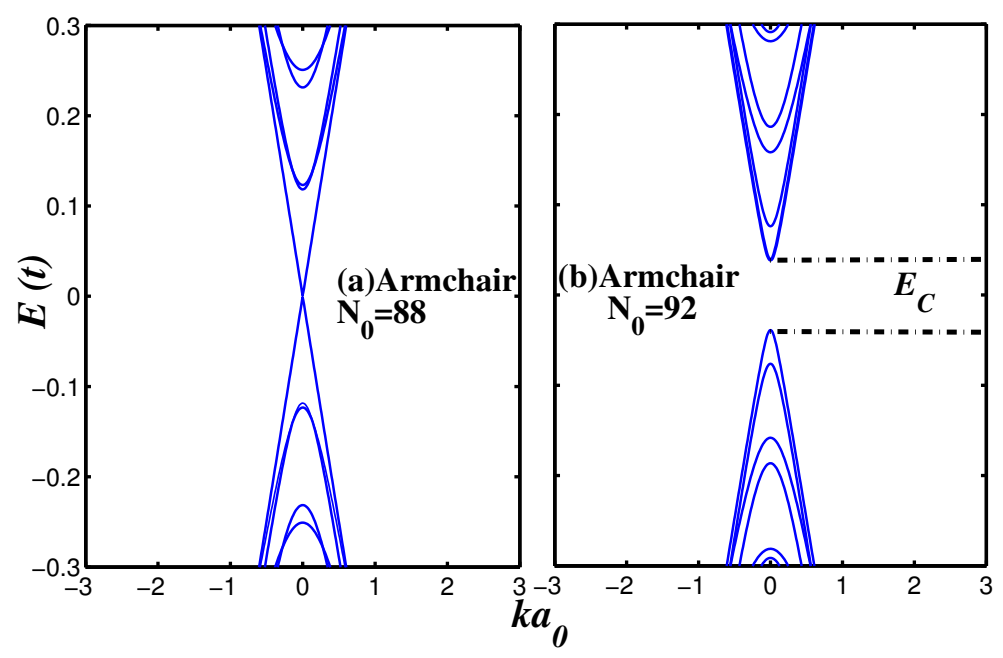

Fig. 3. The band structures for a armchair graphene nanoribbon: (a) $N_{0}=88$, the gap energy is zero, (b) $N_{0}=92$, the gap energy of $E_{C} \approx 0.08 t$ appears [10].

Numerical results fit well with the analytic formula of the gap for armchair GNRs that is given in Ref. [10]:

$$
E_{C}= \pm t\left|2 \cos \left(\frac{p \pi}{n+1}\right)+1\right|
$$

where $\mathrm{t}$ is the nearest neighbor hopping energy $(t=2.7 \mathrm{eV}), \mathrm{p}$ is the quantum number of the subbands, $\mathrm{n}$ is an integer $\left(n=N_{0} / 2\right)$. The gap energy can be inferred from this formula, however, its value varies from particular cases. Furthermore, in the armchair GNRs two Dirac points $\left(K\right.$ and $\left.K^{\prime}\right)$ are mixing together that contribute to one valley exited in the first Brilloin zone compared with two distinct valleys in the zigzag case $[6,8]$. We notice that the dependence of the electronic states on the width of the GNRs can be understood in terms of eigenstates of the Dirac Hamiltonian with appropriate boundary conditions $[1,3,6]$.

Figure. 4 shows the Fermi energy dependence of the conductance for the zigzag and armchair ribbons with $N_{0}=88$ (a) and $N_{0}=156$ (b). It is easy to realize that the ballistic conductance of GNRs increases step by step as a function of the Fermi energy due to the fact that it is simply proportional to the number of the " active transport" channels at a given energy. Each time one channel is opened the conductance is risen by a value of $g_{0} \sim 2 e^{2} / h[1]$. The Fig. 4a shows that the conductance, in the armchair GNRs, increases with the step, $\Delta g=g_{0}$, as a function of Fermi energy. On the contrary, for zigzag GNRs (fig. $4 \mathrm{~b}$ ), the width of the steps in the $G\left(E_{F}\right)$ is two times larger compared to armchair GNRs, $\Delta g=2 g_{0}$. This is resulted from the band structures of GNRs. In the armchair GNRs, due to the mixing two Dirac points, there is only one valley in the first Brilloin 


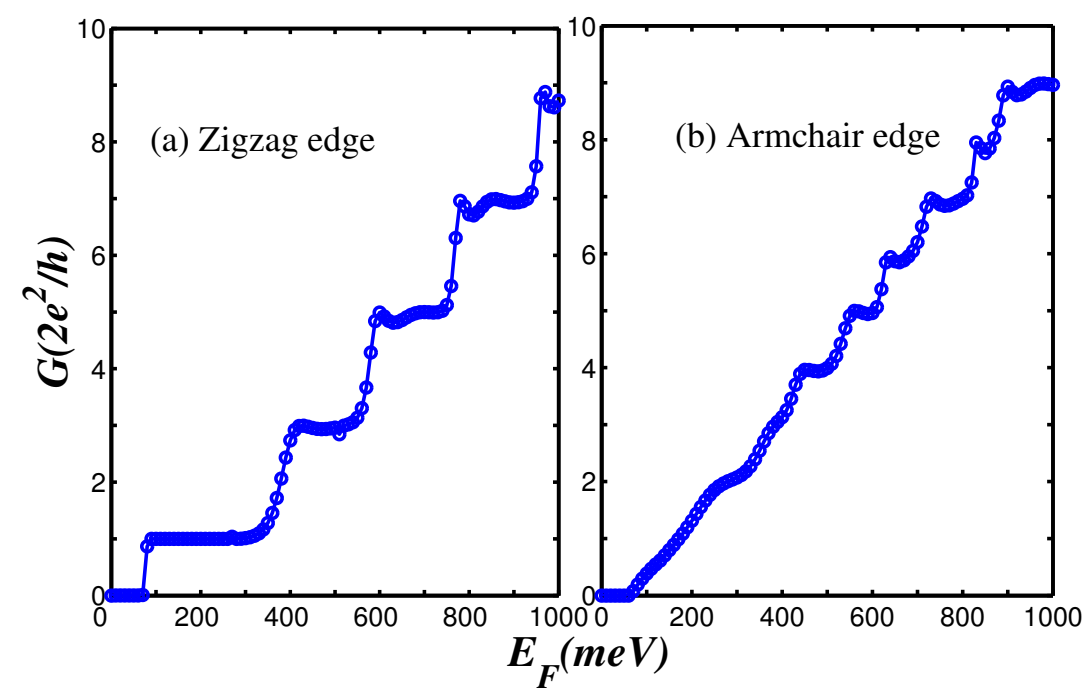

Fig. 4. The conductance as a function of the Fermi energy: (a) armchair and (b) zigzag edges

zone. It means that merely one after another new "active transport" channel is opened as the Fermi energy increases. However, in the zigzag GNRs, there are the two new channels opened simultaneously because of the two valleys in the first Brilloin zone $[3,18,19]$.

In conclusion, we have suggested a tight-binding approach for calculating the band structure and conductance for any periodic $1 \mathrm{D}$ systems. As a useful illustration, numerical calculations have been performed for GNRs with zigzag and armchair edges and with different ribbon widths. Obtained results showed that GRNs are either metallic or insulating depending on their size and geometry and the conductance of GRNs is quantized because of the ribbon finite widths. The suggested TB-approach is quite general and should be applicable to calculate various static and dynamic characters for a wide range of $1 \mathrm{D}$ systems.

Acknowledgements. Authors would like to thank Nguyen Van Lien for suggesting the problem and critically reading the manuscript. This work is supported by the Ministry of Science and Technology via the Fundamental Research Program (Project 4.023.06).

\section{REFERENCES}

[1] A.H.Castro Neto, F.Guinea, N.M.R.Peres, K.S.Novoselov and A.K.Geim, arXiv:0709.1163v1 [condmat.other] 7 Sep 2007.

[2] J.C.Charlier, X.Blas and S.Roche, Rev. Mod. Phys, 79 (2007) 667.

[3] P.G. Silvestrov and K.B. Efetov, Phys. Rev. Lett, 98 (2007) 016802. 
[4] E. B. Sonin, Phys. Rev. B, 77 (2008) 233408.

[5] D.P.DiVincenzo and E.J.Mele, Phys. Rev. B, 29 (1984) 1685.

[6] L.Brey and H.A.Fertig, Phys. Rev. B, 73 (2006) 235411.

[7] M. I. Katsnelson, arXiv:0703190v2 [cond-mat.mes-hall] 30 May 2007.

[8] Yu. Klymenko and O.Shevtsov, arXiv:0806.4531v1 [cond-mat-hall] 27 Jun 2008.

[9] Y.Y.Zhang, J.P.Hu, X. C. Xie, and W. M. Liu, arXiv:0708.2305v1 [cond-mat.mes-hall] 17 Aug 2007.

[10] H.Zheng, Z.Wang, T.Luo, Q.Shi and Jei Chen, Phys. Rve. B, 75 (2007) 165414.

[11] T. C. Li and S.P.Lu, Phys.Rev.B, 77 (2008) 085408.

[12] J. Fernandez-Rossier, J. J. Palacios and L.Brey, Phys. Rev. B, 75 (2007) 205441.

[13] H.Xu, T. Heinzel, M. Evaldsson and I. V. Zozoulenko, Phys. Rve. B, 77 (2008) 245401.

[14] H.Raza and E.C. Kan, Phys. Rev. B, 77 (2008) 245434.

[15] Y.W.Son, M.L. Cohen, and S.G. Louie, Phys. Rev. Lett, 97 (2006) 216803.

[16] L.Yang, C.H.Park, Y.W.Son, M.L. Cohen, and S.G. Louie, arXiv:0706.1589v1 [cond-mat.mes-hall],11 Jun 2007.

[17] E.R.Mucciolo, A.H.Castro Neto, and C.H.Lewenkopf, arXiv:0806.3777v1 [cond-mat.mes-hall] 23 Jun 2008.

[18] Y.M.Lin, V.Perebeinos, Z.Chen, and P.Avouris, arXiv:0805.0035v2 [cond-mat.mes-hall] 1 May 2008.

[19] N.M.R.Peres, A.H.Castro Neto, and F.Guinea, Phys. Rev. B, 73 (2006) 195411.

[20] J.H. Davies, The Physics of low-dimensional semiconductors (Cambridge University press)( 1996)

Received 18 August 2008. 